\title{
Statistical Physics for Cosmic Structures
}

\author{
Luciano Pietronero $^{\mathrm{a}}$, Andrea Gabrielli ${ }^{\mathrm{a}}$, and Francesco Sylos Labini ${ }^{\mathrm{b}}$ a \\ aNFM Sezione Roma1, Dip. di Fisica, Universitá "La Sapienza", \\ P.le A. Moro, 2, I-00185 Roma, Italy. \\ ${ }^{\text {b}}$ Laboratoire de Physique Theorique, Unversité de Paris XI, 91405 Orsey Cedex France
}

Ideas of Statistical Physics are very relevant for cosmic structures especially considering that the field is undergoing a period of exceptional development with many new data appearing on a monthly basis. In the past years we have focused mostly on galaxy distributions and their statistical properties. This led to an interesting debate which will be resolved by the next generation of data in a couple of years. In addition here we discuss the statistical properties of the fluctuations of the cosmic microwave background which are small in amplitude but complex in structure. We finally discuss the connection between these observations and the Harrison-Zeldovich spectrum and its further implications on the theories of structure formation and the and the cosmological $N$-body simulations.

\section{Introduction}

From the point of view of Statistical Physics cosmic structures give rise to a puzzling and apparently contraddictory situation: on one hand the Cosmic Microwave Background Radiation (hereafter CMBR) is extremely isotropic with temperature fluctuations of the order $\delta T / T \simeq 10^{-5}$ [1,7]. On the other hand matter distribution is extremely clumpy: there are galaxies, clusters, filaments and large voids up to huge scales [4, 14]. Fluctuations with a large density contrast $\delta \rho / \rho \gg 1$ appear to characterize the matter field over a broad range of scales. The link between the two observations is, of course, not direct. The first one refers to radiation which is essentially unchanged from the time of the electro-magnetic decoupling. This is when the universe was about 1000 times younger than now. Galaxies instead correspond to the present (visible) matter field. Another important difference is that the CMBR corresponds to an angular distribution, while the galaxy distribution is three-dimensional, in view of the red-shift $(z)$ measurement. Before the decoupling time, matter and radiation are coupled by the Sachs-Wolf effect [12 which describes the fact that if radiation escapes from a gravitational potential well it is red-shifted by an amount linear with the potential.

This implies that, at $z=1000$ (Universe 1000 times younger than now) $\delta \rho / \rho \simeq \delta T / T \simeq$ $10^{-5}$. The question is then if gravitational instability can lead to the present large structures, starting from $\delta \rho / \rho \simeq 10^{-5}$ at $z=1000$. The answer is no, in fact one could get basically an amplification of a factor $10^{3}$ by gravity, in the linear regime, leading to a present value of the matter fluctuations $\delta \rho / \rho \simeq 10^{-2}$, in sharp contrast with the large 
structures observed. This is one of the main reason to introduce a vast amount of nonbaryonic dark matter matter (it interacts very weakly with radiation) which is supposed to have, at the decoupling time, fluctuations much larger than $10^{-5}$. In view of this and other puzzling observations the current interpretation is that the matter field consists in about $99 \%$ of the total matter and the baryonic matter is just about $1 \%$.

From this discussion it is clear that the understanding of the nature of the various cosmic structures and their dynamical development is a central point of the field which has implications in essentially all other properties. Many new data have been generated in the past few years and many more are expected in the near future. So the field is quickly evolving from a situation with many speculations and few data to a new one, in which the new abundance of data permits a test of long standing conjectures. This is therefore an extremely interesting moment in which ideas from Statistical Physics can play a crucial role. This is true grasping the properties of structures from the data but also in the theories of structures formation and in the $N$-body simulations.

In the past we have mostly considered the properties of the galaxy distributions [0, [14]. Here we start instead from an analysis of the fluctuations corresponding to the microwave background radiation, and then we try to connect them to the galaxy properties. We outline the main open problems from a new perspective and we consider some consequences also on the theories and cosmological $N$-body simulations [3],2].

\section{Small fluctuations and Poisson distribution}

The standard approach in the field is to assume that the matter field $\rho(\vec{r})$ is always characterized by a well defined average density with small amplitude fluctuations. In our opinion this may be acceptable in relation to the microwave radiation, but not for the galaxy distribution. We will come back on this point later.

The basic assumption is to have

$\rho(\vec{r})=\rho_{0}+\delta \rho(\vec{r})$ with $\delta \rho(\vec{r}) \ll \rho_{0}$,

where $\rho_{0}$ is the average density. This is then analyzed in terms of the correlation function of the fluctuations (positive and negative)

$\xi(r)=\frac{\left\langle\rho\left(\vec{r}_{0}\right) \rho\left(\vec{r}_{0}+\vec{r}\right)\right\rangle}{\rho_{0}^{2}}-1 \equiv \frac{\left\langle\delta \rho\left(\vec{r}_{0}\right) \delta \rho\left(\vec{r}_{0}+\vec{r}\right)\right\rangle}{\rho_{0}^{2}}$

the normalized mass variance in spheres of radius $R$

$\sigma^{2}(R)=\frac{\left\langle M^{2}(R)\right\rangle}{\langle M(R)\rangle^{2}}-1$,

(where $M(R)$ is the mass in a given sphere of radius $R$ ) and the power spectrum of the normalized density fluctuations which are supposed to have small amplitude with respect to the average density

$P(k)=\left\langle|\hat{\delta} \rho(\vec{k})|^{2}\right\rangle=\frac{1}{(2 \pi)^{3}} \int d^{3} r e^{-i \vec{k} \vec{r}} \xi(r)$,

where $\hat{\delta} \rho(\vec{k})$ is the Fourier transform of the normalized density contrast field $\delta \rho(\vec{r}) / \rho_{0}$. 
It is very instructive to consider various properties and in particular the fluctuations of the gravitational potential corresponding to a Poisson distribution of mass points. The Poisson distribution is completely random and uncorrelated. In a sphere of radius $R$ we have an average number of points given by

$$
\langle N(R)\rangle=\rho_{0} V \sim \rho_{0} R^{3},
$$

where $V \sim R^{3}$ is the volume of the sphere in three dimensions. The typical number fluctuation in such a sphere is given by

$\delta N(R)=\langle N(R)\rangle^{\frac{1}{2}} \sim R^{\frac{3}{2}}$

With respect to our functions we have that in the thermodynamic limit of an infinite distribution of points

$\xi(r)=\delta(\vec{r}) ; P(k) \sim k^{0} \sim$ const. $>0$.

However in a finite sample, for the finite size fluctuations due to Eq.(6), for a generic statistical estimator of $\xi(r)$ we have

$|\xi(r)| \simeq\left|\frac{\tilde{\delta \rho}}{\rho_{0}}\right| \simeq\left|\frac{\delta N}{\langle N\rangle}\right| \simeq\langle N\rangle^{-\frac{1}{2}} \sim r^{-\frac{3}{2}}$

for the absolute value of $\xi(r)$, where $\tilde{\delta} \rho$ is the typical fluctuation of the conditional density from an occupied point $\mathrm{For} P(k)$ the finite value of $N$ induces some noise on top of the constant value of Eq.(7).

Now comes the key-point because we can estimate the fluctuations of the gravitational potential $\delta \phi(R)$ as a function of the scale $R$ :

$\delta \phi(R) \sim \frac{\delta N(R)}{R} \sim R^{\frac{1}{2}}$

So we have diverging fluctuations of the potential originated by the finite size fluctuations of the Poisson distribution Eq.(6). This is a very important point which has deep implications with respect to the Harrison-Zeldovich spectrum (see below). However, if we estimate in the same way the fluctuations of the gravitational force $\vec{F}(R)$ we have the surprising result that they are instead bounded

$|\vec{F}(R)| \sim|\nabla \delta \phi(R)| \sim R^{-\frac{1}{2}}$.

This result, that the gravitational force is well defined in a Poisson distribution of points, is well known since the seminal work of Chandrasekhar [5]. The extension of this result to the fractal case is rather complex, and the interested reader can find a discussion in [9].

These properties of the gravitational field of a random distribution of of massive points are usually not very appreciated but they already pose, in our opinion, extremely interesting and challenging problems as we are going to see in the following.

\footnotetext{
${ }^{1}$ Note that there are some special statistical estimators of $\xi(r)$, for the particular case of a Poissonian distribution only, giving $|\xi(r)| \sim r^{-3}$.
} 


\section{The Harrison-Zeldovich (HZ) spectrum}

We consider now the standard derivation of the $\mathrm{HZ}$ spectrum of primeval fluctuations which has a central role in the field. The idea is to consider the implications of the CMBR observations as given for example by the COBE experiment [1]. In this experiment the temperature fluctuations of the black body spectrum are measured at large angular separation ranging from 7 to 90 degrees. The observation is that the value $\delta T / T \simeq 10^{-5}$ is about the same for small and large angles. This means that this fluctuation does not grow with the angle. The standard conjecture (i.e. 12]) is that one can go from angles to distances and argue that

$\delta T(\theta) \sim \delta \phi(R) \sim$ const.

Namely, if the temperature fluctuation is angle independent, then the gravitational potential fluctuation which, via the Sachs-Wolf effect led to the temperature fluctuation, should be independent on the scale. This is sometimes defined as a conditional "scale invariance", but instead is just that the potential fluctuations are simply constant at various scales [8]. We can immediately see that a Poisson distribution does not satisfy this condition because the fluctuations of the gravitational potential grow with scale as in Eq.(9). It is easy to see that, in order to satisfy the Eq.(11), one should have

$\delta N(R) \sim\langle N(R)\rangle^{\frac{1}{3}}$,

which implies a more regular distribution (super-homogeneous) with respect to a random Poisson one (see Fig. (1). With respect to the correlation function and the power spectrum, it can be easily shown that at large scales [8]

$$
\begin{aligned}
\xi(r) & \sim-r^{-4} \\
P(k) & \sim k .
\end{aligned}
$$

Note the negative sign for the large scale behavior of $\xi(r)$ : this means anti-correlations at large scales (see Fig. 2).

This discussion leads to a series of questions about the implications of the density fluctuations on the gravitational potential which, in our opinion, would require some deeper clarifications: (i) a Poisson distribution has a perfectly well defined average density at large scales $\rho_{0}=\lim _{V \rightarrow \infty} N(V) / V$. Consequently, one could suppose that at large scale one can use the value $\rho_{0}$ in general relativity and describe it in terms of the Friedman metrics. However, given our discussion and Eq.(9), this should not be permitted because the finite size fluctuations $\delta N \sim\langle N\rangle^{1 / 2}$ induce diverging gravitational potential fluctuations even at large scales where $\delta N \ll\langle N\rangle$. If, on the other hand, one would compare forces and not potentials, then the perturbation would be negligible. So the question to clarify is whether a Poisson distribution of mass points is compatible with a Friedman cosmology at large scales. (ii) The same reasoning holds for the derivation of the HZ spectrum as necessarily implied by the observed properties of the CMBR. Note that the HZ spectrum is so central to the field that this and the previous points should either be reinforced on disproven. (iii) Other points concern the relations between angular and space correlations. We have learned that for galaxies the two properties can be very different due to the projection effects of the angular case. Finally the Sachs-Wolf effect for non trivial density fluctuations should be considered. 

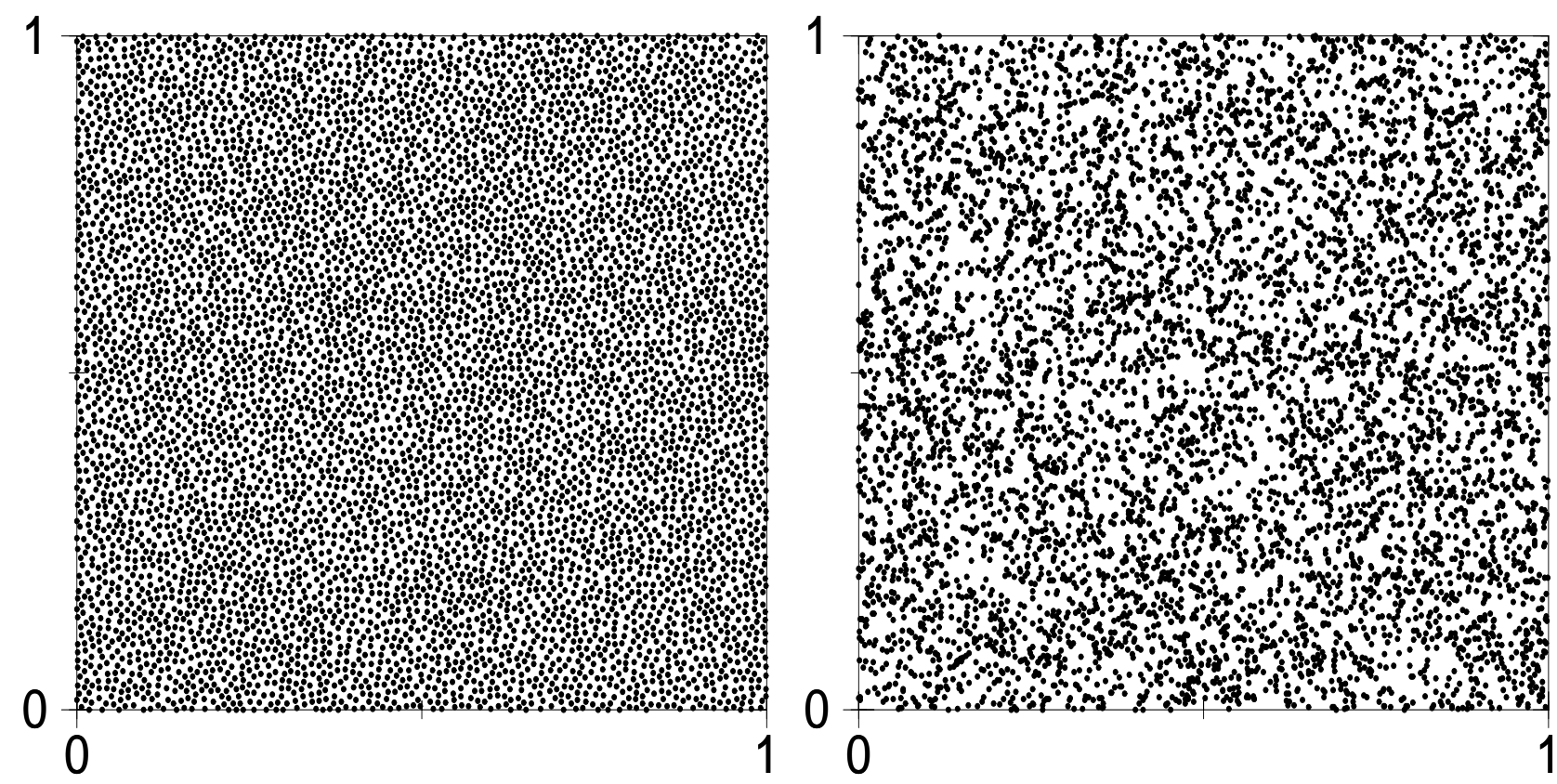

Figure 1. Left panel: Glass-like distribution of points obtained by the method of the shuffled lattice [8]. The idea is to decrease the fluctuations below to the level of the Poisson random distribution. This achieved by imposing a maximal value for the nearest neighbors distance. In this case the fluctuation is about $\delta N(R) \sim\langle N(R)\rangle^{1 / 3}$ and it is a qualitatively good representation of the HZ spectrum which in this respect can be considered superhomogeneous because it is more homogeneous than a Poisson distribution. Right panel: Example of a Poisson distribution. In this case the behavior of the typical fluctuation of the number of points in a ball of radius $R$ goes like $\delta N(R) \sim\langle N(R)\rangle^{1 / 2}$. The distribution is homogeneous at large scale with a well defined average density.

\section{Galaxy correlations updated}

For galaxy correlations all the above concepts of small fluctuations theory etc. are certainly not appropriated. One observes fractal clustering with large scale structures and voids for which $\delta \rho / \rho_{0} \gg 1$ or even worse $\rho_{0}$ is not well defined in the available samples. This is the field in which we have worked extensively and proposed a radically new and more general way to interprete the puzzling observations and to define the theoretical perspective. One of the consequence of our work is that the so called characteristic length of galaxies $r_{0} \simeq 5 \mathrm{Mpc} / \mathrm{h}$ [12 is an artefact of the a-priori assumption of homogeneity and, in our opinion, correlations extend up to the present limits of observations [4, 14, 11]. In any case there is, finally, agreement on the fact that at least to some small scale, galaxy correlations are fractal. A common misunderstanding is however to think that if there would be a crossover, then the small fluctuations theory could describe the fractal properties because they extend only on a finite range of scale (e.g. [15]). This is clearly incorrect because, independently of the presence of the crossover, a theory based on small fluctuations with $\delta \rho / \rho_{0} \ll 1$ can never be appropriated to describe fractal clustering. 

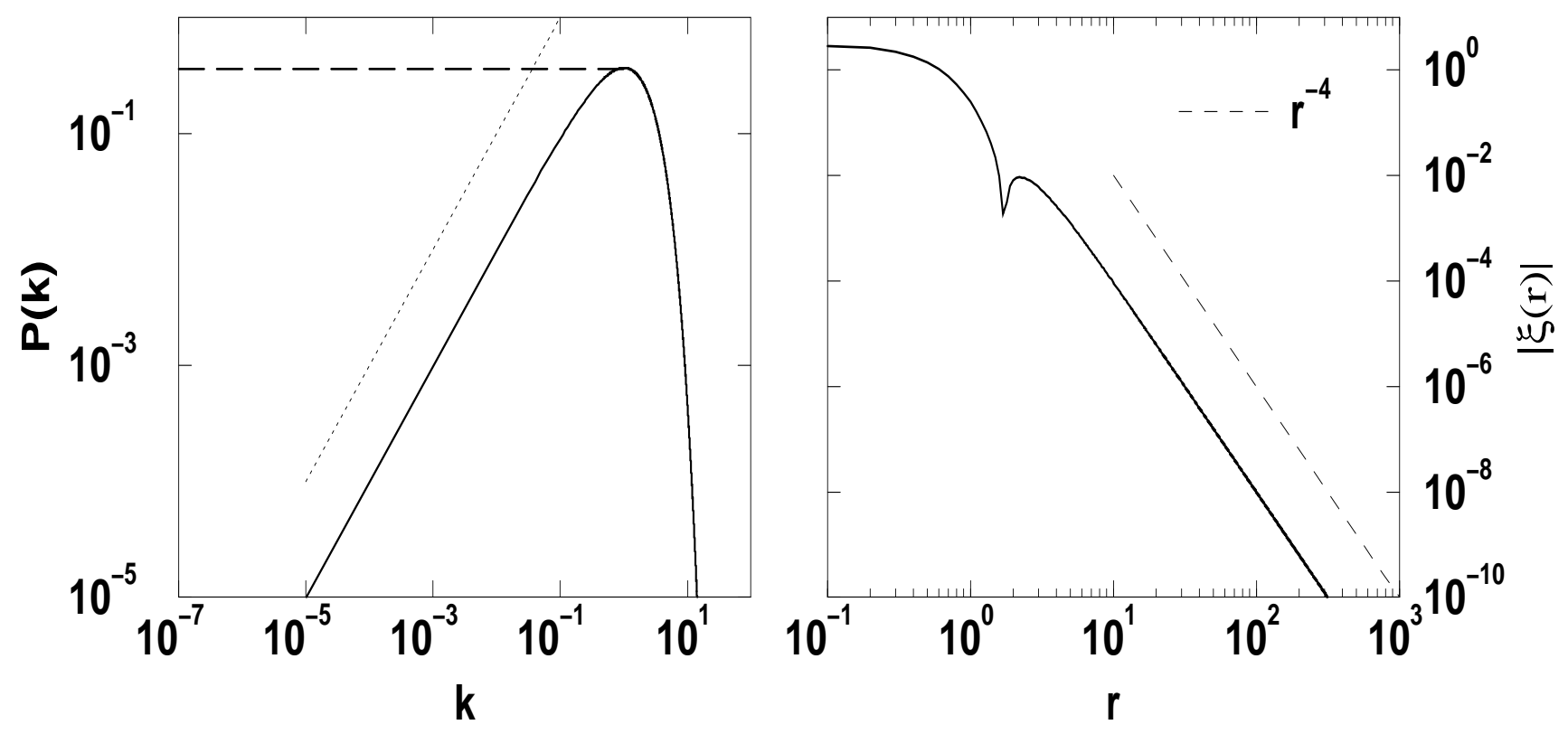

Figure 2. Left panel: Power spectrum corresponding to the HZ spectrum (solid line). Note that the behavior at large scale (small $k) P(k) \sim k$ (dotted line) implies less fluctuations than the Poisson behavior $P(k) \sim k^{0} \sim$ const. $>0$ (dashed line). Right panel: Modulus of the correlation function $\xi(r)$ (solid line) corresponding to the previous power spectrum. Note that at large scales the function is negative (the reference dotted line has a slope $\left.r^{-4}\right)$. Note that the units are arbitrary.

Apart from this conceptual point, which is anyhow valid, the question of the extension of the fractal properties should be clarified by next catalogues. In the next few years the new catalogs like $2 \mathrm{dF}$ and SDSS will dramaticaly improve our knowledge of the large scale distribution of galaxies. The ongoing debate in the field [6, 13, 15, 11] will be then clarified by the analysis of these new data.

It is clear therefore that, at least for the properties of galaxy clustering, the formalism of the small fluctuation theory is not appropriate at any stage from the data analysis to the formulation of a theory of structure formation.

\section{Conclusion and perspectives}

We have presented a brief overview of the relevance of Statistical Physics for cosmic structures. Many new data are appearing for the CMBR and for the galaxy distribution, and modern Statistical Physics is the natural framework to identify the relevant structures and defining the corresponding theoretical models.

In this respect the situation appears quite different for the very small fluctuations in the CMBR and the very large fractal fluctuations observed for galaxies. In the first case the traditional theory of small fluctuations could be appropriated, but it is certainly not appropriated for galaxies. Whatever clustering is observed this has scaling and fractal 
properties and, even if there would be a crossover to an homogeneous distribution (a fact which is still far from shown) the fractal clustering could never be properly described by a small amplitude fluctuations theory.

A different question concerns the cosmological model able to describe a situation where matter has fractal properties. In Ref. [10] the interested reader can find an attempt to construct a Friedman model with zero density, where the fractal can be treated as perturbation to the background homogeneous metric.

It is therefore unavoidable to generalize the approach to include the possibility of complex structures and large fluctuations at alla levels, from the data analysis to the theoretical methods and the $N$-body simulations [3,2]. This should lead to a new general perspective of the field, strongly based on a cross-disciplinary approach, which certainly will lead to novel exciting developments.

\section{REFERENCES}

1. Bennett C.L. et al. Astrophys.J., 436 (1994), 423

2. Baertschiger T. \& Sylos Labini F., Preprint (2001) astro-ph/0109199

3. Bottaccio M. et al.preprint 2001

4. Coleman, P.H. \& Pietronero, L., Phys.Rep. 231, (1992) 311

5. Chandrasekhar S., Rev. Mod. Phys. 15, 1 (1943)

6. Davis, M., in the Proc. of the Conference "Critical Dialogues in Cosmology" N. Turok Ed., p.13 (1997), World Scientific

7. De Bernardis P. et al Nature (2001)

8. Gabrielli A., Joyce M. and Sylos Labini F. Preprint (2001)

9. Gabrielli A., Sylos Labini F. \& Pellegrini S., (1999) Europhys.Lett. 46, 127

10. Joyce M., Anderson P. W., Montuori M., Pietronero L. and Sylos Labini F., (2000) Europhys.Letters 50, 416-422

11. Joyce M. \& Sylos Labini F., Astrophys. Journal Letters 554, L1-L4, (2001)

12. Peebles, P.E.J., Principles of Physical Cosmology, Princeton Univ. Press, (1993)

13. Pietronero, L., Montuori, M. and Sylos Labini, F., in the Proc of the Conference "Critical Dialogues in Cosmology" N. Turok Ed., p.24 (1997), World Scientific

14. Sylos Labini F., Montuori M. and Pietronero L., Phys.Rep.,293 (1998),66

15. Wu K.K., Lahav O.and Rees M., Nature, 225, (1999) 230 